\title{
An Overview of Semantically-Based TCM Telemedicine Systems
}

\author{
${ }^{1}$ HerbMiners Informatics Limited, Unit 209A, 2/F Photonics Centre, Phase One, \\ Hong Kong Science and Technology Park, Pak Shek Kok, Tai Po, NT, Hong Kong \\ \{iamjackei, wilfred.lin\} @gmail.com \\ ${ }^{2}$ Department of Computing, The Hong Kong Polytechnic University, \\ Hung Hom, Kowloon, Hong Kong \\ \{iamjackei, wilfred.lin\}@gmail.com
}

\begin{abstract}
Traditional Chinese Medicine (TCM) is gaining its popularity in recent years. The limited exchange of medical knowledge due to geographical constraints etc. will be disadvantageous to patients. It is crucial to capture the knowledge and experience in a computer-based representation. With the application of semantic network and ontology, knowledge and experience will then be kept and stored in a semantic manner, which facilitates effective and efficient knowledge exchange and sharing.
\end{abstract}

Keywords: Semantic network $\cdot$ Ontology $\cdot$ Telemedicine $\cdot$ Traditional Chinese Medicine

\section{Introduction}

The knowledge of Traditional Chinese Medicine (TCM) has existed for more than 3000 years till now, while TCM practices are in various forms according to different geographical and environmental constraints. The exchange of medical knowledge and clinical experience was limited due to transportation difficulties, language barriers etc. As times go by, the stored medical information has been gradually refine to constitute more than 5000 pieces of works in extant. Useful herbal ingredients including plant parts, minerals and animal matter have been added and incorporated into the conceptual TCM pharmacopeia.

Traditional Chinese Medicine (TCM) has been widely used for many centuries in China, it is gaining more and more adoption all over the world. The trends in the use of TCM will be aided by standard and uniform vocabulary and an agreed consensus by the domain expert, it is crucial to capture the underlying clinical TCM body of knowledge and practices in a computer-based representation. This has a semantic basis in which the focus is on the meaning of the knowledge but not the syntactic format of its representation. Ontology will be utilized as well to make the knowledge more readily searchable and queried.

\section{TCM and Telemedicine}

One of the earliest definitions of the notion of Telemedicine can be found in the publication [Lacroix99]. It refers to make use of the Internet to deliver medical care to every corner all over the world. Thus, those web-based medical system has at least 
some telemedicine elements, independent of its scale. Telemedicine systems can be broadly divided into different types, according to their goals and functions as follows:

a) Curative Systems - medical practitioners use the system to achieve the diagnosis and treatment goals.

b) Consultative Systems - through the system interface the user can obtain needed information, such as information about named patent drugs, and addresses/expertise of medical practitioners in the region/vicinity.

c) Medical Information Management Systems in terms of data storage and retrieval (e.g. medical images).

d) Decision Support Systems - this happens in various forms, for example, the physician, who is using a telemedicine system (e.g. the PuraPharm's D/P (diagnosis/prescription) system in a mobile clinic [JWong09] may import biometric reports to aid the diagnostic decision/precision as shown by Fig. 1.

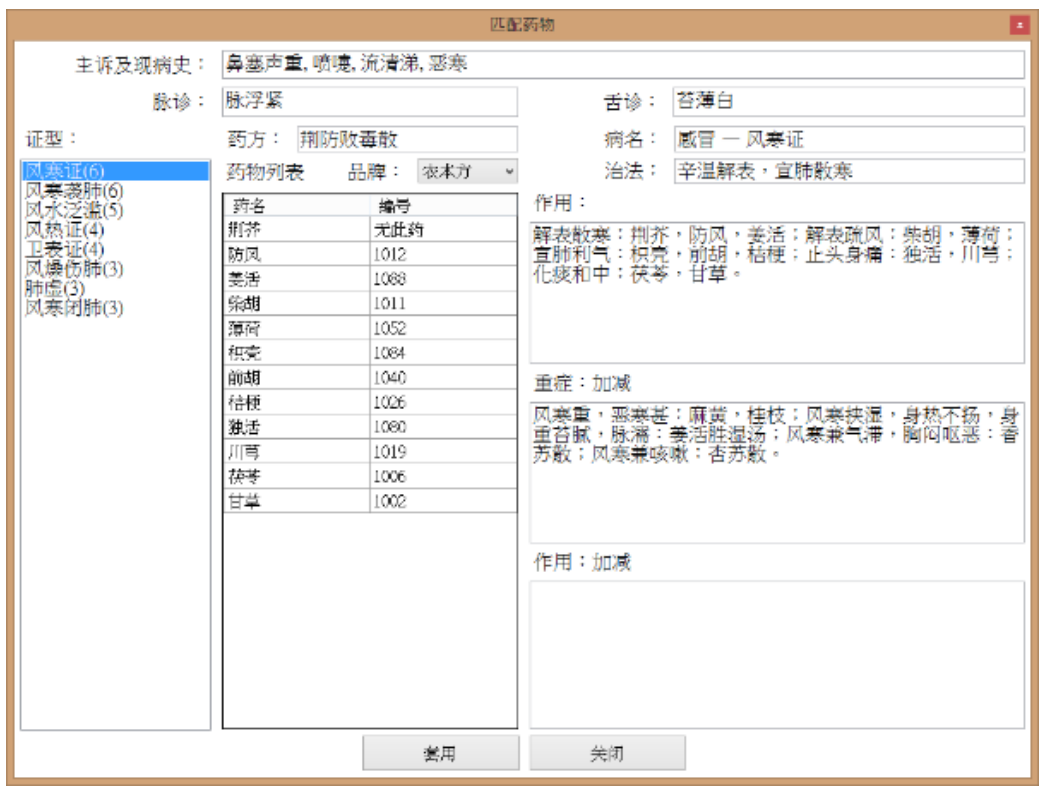

Fig. 1. Imported information to aid diagnosis

The aim of doing this is to let people including non-medical domain expert and registered medical personnel consult and verify knowledge if necessary from the knowledge base. Yet, a knowledge base can be for a general purpose and similar to a dictionary in this sense. It also help experts to clarify doubts due to multirepresentations owing to disparate national or regional conceptions of a medical phenomenon. A typical example in the Western/allopathic medicine area is the UMLS (Unified Medical Language System [UMLS]), which was developed by the US National Library of Medicine to resolve the differences in Western/allopathic clinical terminology due to regional and/or national disparities. The UMLS is ontology-based. Another ontology-based system is the disease and treatment ontology for Western Allopathic Medicine [Hadzic05 and Hadzic10]. By its nature the UMLS is not for 
frontline clinical application to allow computer-aided diagnosis and treatment in contrast to the PuraPharm's TCM (Traditional Chinese Medicine) telemedicine mobile clinic (MC) system. The UMLS is consultative in nature. That is, it is not a clinical system but a consultation setup that people can interact with to sort out terminology problems. Therefore, its aim is to provide an interactive learning, reference, and bridging mechanism for knowledge gaps in a global sense. For example, in conventional/Western/allopathic medicine different countries may have different definitions for an observed phenomenon. In order to resolve the similarity and differences of various definitions, as well as language peculiarities on a global scale, meta-thesauri can serve as an effective means as shown by the UMLS.

\section{Explicit and Implicit Semantics}

If a single statement composed of a string pattern which can induce various interpretations for different people with different perspectives, the semantics of this string is then considered implicit. This is similar to deciphering the embedded logic in a given program without its original detailed specification. The logical representation can vary from one interpreter to another. For example, the hierarchy represented in the UML (Unified Modeling Language) style in Fig. 2 can result in ambiguous interpretations (i.e. semantics) from different people for the "logical points" a and b, which can be logically AND or OR; vice versa; or EXLUSIVE OR, leading to unexpected results. For curative medicine, this kind of logical freedom is disadvantageous to the patients. For this reason the semantics in the ontology must be explicitly defined by the principle of "single meaning for every semantic path".

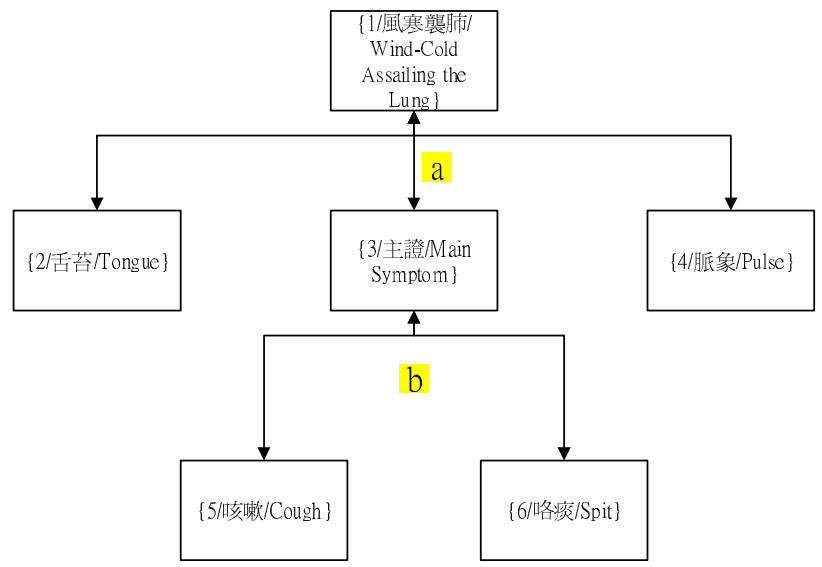

Fig. 2. Implicit logical representation - implicit semantics (bilingual) 


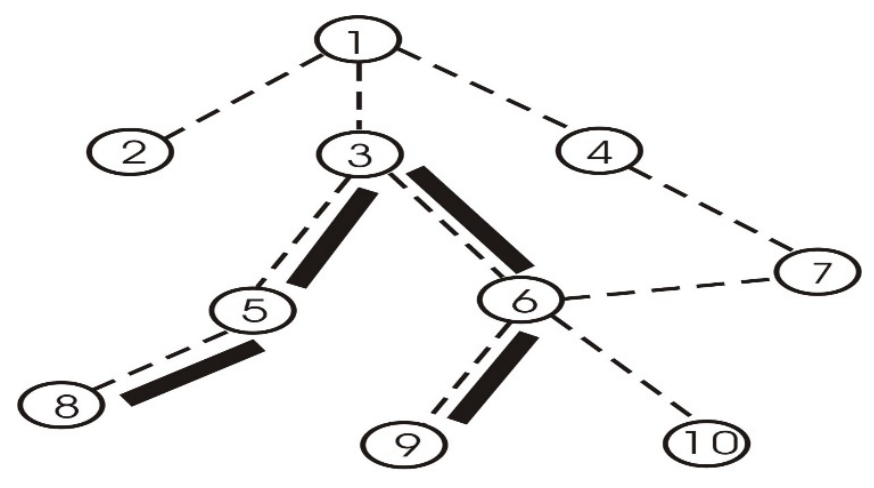

A complete tree

Fig. 3. Semantic paths in a 3-dimensional semantic subsumption hierarchy

The 3-dimensional subsumption hierarchy in Fig. 3 contains many operations or semantic paths (e.g. $\{3,5,8\}$ and $\{3,6,9\}$ ). Yet, from a 2-dimensional viewpoint Fig. 2 is simply a network representation of many possible operational paths (i.e. traversal paths) between any two points. To create an operational ontology-based system (with a mini-ontology) out of this tree, it is necessary to elaborate this 2-dimensional view into a 3-dimensional one. Then, the focus is represented by the chosen "lead", which in reality is the first point where the parsing mechanism begins to process. For example, if node 1 is the chosen lead for parsing in Fig. 3, then the possible 14 semantic paths for the 3-dimensional subsumption hierarchy are the following: $\{1,2\} ;\{1,3\}$; $\{1,3,5\} ;\{1,3,5,8\} ;\{1,3,6\} ;\{1,3,6,9\} ;\{1,3,6,10\} ;\{1,4\} ;\{1,4,7\} ;\{1,4,7,6\}$; $(1,4,7,6,9\}$; and $\{1,4,7,6,10\}$. If every semantic path is supported by only a single dedicated software module/object, then the meanings of the set of semantic paths (represented by the equal number of software modules or objects) form the lexicon for this particular system. As a result, the system always gives a clear, consistent and unambiguous (i.e. explicit) answer to every query, which is another form of a semantic path in the lexicon. Although the semantic paths in the lexicon are explicit, every query is constructed "implicitly" or with user-transparency according to the input sequence of the atomic elements. In fact, the semantic paths can be further classified into more specific semantic groups for various goals, similar in fashion to the conceptual UMLS framework.

\section{Ontology-Based TCM Telemedicine System - Major Aspects}

The focus of this paper is to give an overview of the semantic TCM telemedicine system. The following aspects should be addressed:
a) Ontology Modelling
b) Ontology Implementation Tool
c) Internet Capability 
d) $\quad$ Cross-layer Logical Transitivity
e)
f)
g)

\subsection{Ontology Modelling}

The design of the system begins with the ontology model blueprint layout followed by the consensus certification. The ontology includes the whole or part of the formalisms and knowledge in the relevant domain. In this sense, an ontology-based system is data/knowledge oriented. This model should be understandable by both TCM domain experts and system developers so as to facilitate evaluation and critiquing of the ontology by the domain experts for correctness and completeness.

\subsection{Ontology Implementation Tool}

The tool is usually a high level language, which can correlate all the concepts in the ontology into a logical subsumption hierarchy. The ontology modelling blueprint is mainly for human understanding, and the embedded subsumption hierarchy should be translated meticulously into the corresponding semantic network for machine understanding/processing/execution. Therefore, it is necessary to choose a tool that has the support of "ontology model blueprint-to-semantic-net translation". It is better for the conversion process to be automatic [JWong08b]. In this light, the languages (or metadata models/systems) proposed by W3C (World Wide Web Consortium) [W3Ca, W3Cb], namely, XML (Extensible Mark-up Language), RDF (Resource Description Framework), and OWL (Web Ontology Language) are good to choose from, as they have widespread automatic translation support.

\subsection{Internet Capability}

Telemedicine relies on the Internet to achieve different goals on the web. For example, the telemedicine system may send out analysts or data-miners to search the web for necessary information to support the system's ontology evolution - the concept of a living ontology [JWong08a, WLin13, AWong15]. This is well exemplified by the $2^{\text {nd }}$ generation of the PuraPharm D/P (diagnosis/prescription) telemedicine system that supports the YOT (a charitable organization in Hong Kong) mobile clinics in Hong Kong. These mobile clinics have been treating thousands of patients weekly in recent years.

\subsection{Cross-Layer Logical Transitivity}

A practical telemedicine system should have a 3-layer architecture. These three layers are as follows: 
- Bottom Layer - the knowledge/database that embeds the subsumption hierarchy that represents the logical relationships among the physical data items/entities included in the consensus-certified ontology.

- Middle Layer - this subsumption hierarchy is also realized in the middle layer as the semantic network for machine understanding and execution.

- Top Layer - the query system that implements the ontology for user understanding and manipulation.

The three layers are logically clones of one another, and therefore they should have cross-layer semantic transitivity. With this transitivity any entity in any layer should have corresponding representations in the other two layers.

It is useful to compare the TCM Curative and Decision Support System with the 3 Layer Architecture of the UMLS consultation system. The UMLS is ontology-based and has three distinctive layers: i) the modularized query system (the modules | are semantic groups) at the top level, ii) the middle logical semantic-net layer, which was constructed from the semantics embedded in the information of the bottom ontological layer, and iii) the bottom ontology is integrated by nature and normally has a subsumption hierarchy of various sub-ontologies of different origins [Gruber93a, Gruber93b, Guarino95].

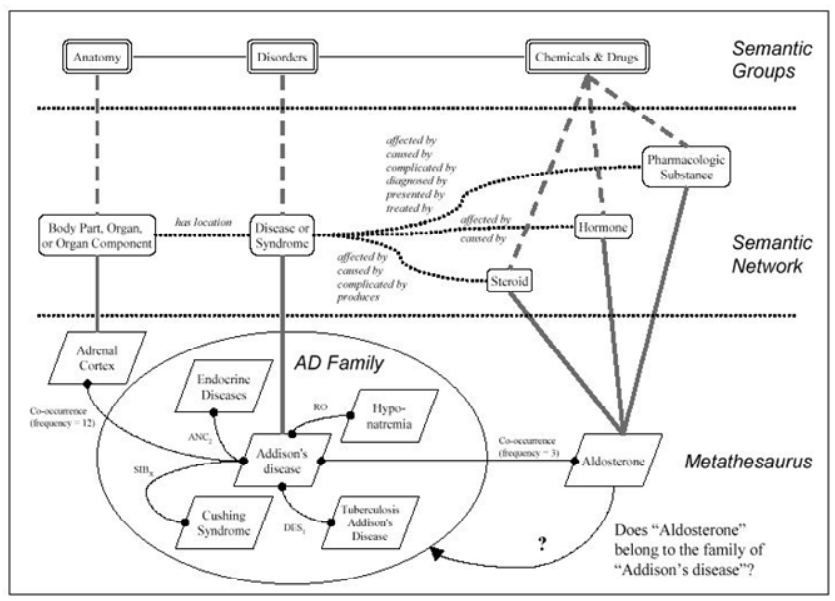

Overview of the methodology applied to the relationships of "Aldosterone" to "Addison's disease"

Fig. 4. The 3-layer UMLS hierarchy [UMLS]

There is, therefore, some similarity between the overall structures of the two systems even though one is used for Curative and Decision Support Purposes whilst this is used as a consultation system. 


\subsection{Automatic System Generation}

Cross-layer semantic transitivity can be achieved correctly by automatic system generation or customization (ASG/C). This approach is a new software engineering paradigm, which requires the user to provide the ontology blueprint layout. With support of the master ontology, where the ontology specification is either a part or the whole of it, the ASG/C mechanism generates/customizes the final ontology-based system in one shot.

\subsection{Pervasive Support}

A telemedicine system requires the support of a wireless-based pervasive computing infrastructure (PCI), which maintains the smart space for the collaborating systems. In the PuraPharm mobile clinic environment, the collaborating systems are the mobile clinics. The essence of the PCI support is better explained by using the successful PuraPharm's mobile clinic (MC) based telemedicine D/P (diagnosis/prescription) system depicted in Fig. 5. The PCI maintains the smart space, which is each occupied by a mobile clinic. The mobile clinic then communicates with the central system, as well as its peers, via the wireless means provided by the PCI. The MC operation is semi-autonomous because the physician can treat the patient at the spot, but the case history of the patient may have to be downloaded from the central computer that runs the fast network. The MC has to inform the central system of its updated local drug inventory. The central system also collects the necessary MC statistics on-line for proactive planning and action. If the MC physician needs help in the diagnostic process, the central system would solicit the relevant information from other friendly sites via the Internet.

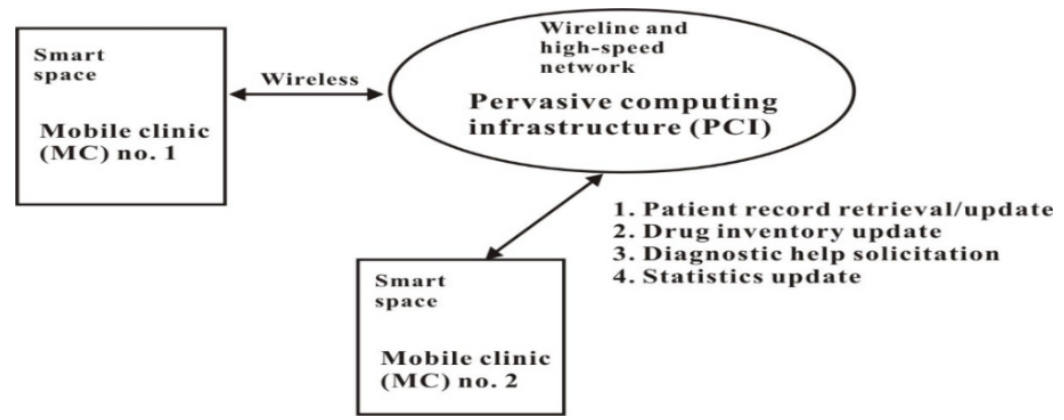

Fig. 5. A PuraPharm's pervasive MC-based telemedicine D/P system

\subsection{Ontology Evolution Approach}

It is important that the TCM ontology keeps itself updated from time to time by taking into account the new knowledge and insights that are being generated as time passes. It should be able to absorb information from every fresh case into the "master" ontology as new knowledge. In this light, this master ontology is a living and evolving system. 
Hence, it is clear that there is a need for a framework of ontology evolution for the TCM ontology so that it reflects the new knowledge and insights. To achieve ontology evolution, we need to:

- Ascertain the new knowledge

- Represent this potential new knowledge temporarily in a form compatible with the ontology

- Carryout consensus certification of this new potential new knowledge

- Enhancing the master ontology only with the consensus certified portions of the new knowledge

In order to ascertain the new knowledge, a text mining approach will be utilized. The data and text mining approach suggests potential changes to the TCM ontology. A temporary representation of these potential changes is stored in a part of the master aliasing table. The proposed potential changes to the ontology are then subject to consensus certification by a panel of TCM domain experts, and only those changes that are approved by this consensus certification are then included into the master TCM ontology through the master aliasing table.

\section{Conclusion}

This paper discussed an overview of semantically-based TCM telemedicine systems. The underlying principles of such a TCM telemedicine system were briefly explored. The proposed future work is to apply the technique of deep learning and predictive analysis for diagnosis/prescription differentiation recommendations.

\section{References}

1. Wong, A.K.Y., Wong, J.H.K., Lin, W.W.K., Dillon, T.S., Chang, E.: Semantically Based Clinical TCM Telemedicine Systems. Studies in Computational Intelligence, vol. 587, pp. 1-152. Springer (2015). ISBN 978-3-662-46023-8

2. Gruber, T.R.: A Translation Approach to Portable Ontology Specification. Knowledge Acquisition 5(2), 199-220 (1993)

3. Gruber, T.R.: Toward principles for the design of ontologies used for knowledge sharing. In: Proceedings of the International Workshop on Formal Ontology in Conceptual Analysis and Knowledge Representation, Padova, Italy, March 17, 1993

4. Guarino, N., Giaretta, P.: Ontologies and Knowledge Bases: Towards a Terminological Clarification. Towards Very Large Knowledge Bases: Knowledge Building and Knowledge Sharing, 25-32 (1995)

5. Hadzic, M., Chang, E.: Medical ontologies to support human disease research and control. International Journal of Web and Grid Service, Inderscience (2005)

6. Hadzic, M., Wongthongtham, P., Dillon, T., Chang, E.: Ontology-based Multi-agent Systems, 274p. Springer Publications, Germany (2010). ISBN 978-3-642-01903-6 
7. Wong, J.H.K., Dillon, T.S., Wong, A.K.Y., Lin, W.W.K.: Text mining for real-time ontology evolution. In: Data Mining for Business Applications, pp. 143-150. Springer (2008). ISBN: 978-0-387-79419-8

8. Wong, J.H., Lin, W.W., Wong, A.K., Dillon, T.S.: An ontology supported meta-interface for the development and installation of customized web based telemedicine systems. In: Brinkschulte, U., Givargis, T., Russo, S. (eds.) SEUS 2008. LNCS, vol. 5287, pp. 233-244. Springer, Heidelberg (2008)

9. Wong, J.H.K., Lin, W.W.K., Wong, A.K.Y., Dillon, T.S.: TCM (Traditional Chinese Medicine) telemedicine with enterprise ontology support - a form of consensus-certified collective human intelligence. In: Proceedings of the International Conference on Industrial Technology (ICIT). Monash University, Victoria, Australia, February 10-13, 2009

10. Lacroix, A., Lareng, L., Rossignol, G., Padeken, D., Bracale, M., Ogushi, Y., Wootton, R., Sanders, J., Preost, S., McDonald, I.: G-7 Global Healthcare Applications Sub-project 4. Telemedicine Journal, March 1999

11. UMLS. http://umls.nlm.nih.gov/

12. W3C, Ontology Definition MetaModel (2005). http://www.omg.org/docs/ad/05-0801.pdf\#search='Ontology\%20Definition\%20Metamodel

13. W3C, Web Service Architecture (Working Paper). http://www.w3.org/TR/ws-arch/

14. Lin, W.W.K., Wong, J.H.K.: Artificial neural network based chinese medicine diagnosis in decision support manner and herbal ingredient discoveries. In: Data Analytics for Traditional Chinese Medicine Research, pp. 123-132. Springer (2012) 